\title{
New Access to Improve Super Resolution using Convolution Neural Network
}

\author{
Rahul Bhattacharya K. Parvathi
}

\begin{abstract}
Super Resolution is the process to enhance image quality by increasing the pixel densities from a low resolution image. Several methods are proposed in the last few decades. We survey several methods like filtration method i.e. Scalar Smoothness Index filtration, learning based method using Convolution Neural Network. We also propose a new algorithm where we use filtration technique as a preprocessing technique of learning based method.

Keywords - Wavelet decomposition technique, SSI(Scalar Smoothness Index), SRCNN (Super-Resolution Convolution Neural Network), PSNR(Peak_Signal-to-Noise_Ratio)
\end{abstract}

\section{INTRODUCTION}

In the field of image processing super-resolution is an important topic which mostly reflects in the application of medical image analysis, agricultural pest detection, CCTV footage analysis for cyber investigations and satellite image enhancement.

Super Resolution (SR) is the process of improving image quality by increasing pixel densities in a Low Resolution (LR) image and obtain a High Resolution (HR) image as an output. There are many SR techniques proposed in last few decades. The popular interpolation techniques such as Bilinear, Bicubic, Lanczos, B-Spline interpolation methods can increase pixel-density but these techniques are not well enough in extraction of edge artifacts. Interpolation technique performs well in smooth region. The SSI-filtration technique is basically a high frequency image filtration technique which can extract the high frequency components i.e. the edge artifacts. The filtration technique mainly increase the contrast of the image, but for the flat region it cannot work satisfactorily. The learning based method is mainly a point-to-point mapping between LR and HR and this prior model of LR and HR image can be mapped with the help of Super-Resolution Convolution-Neural- Network (SRCNN) [16][17]. But this SRCNN method introduced a preprocessing technique only with Bicubic interpolation which draw some disadvantages of loss of artifacts and flatness.

In our paper we approach a new algorithm which can improve the SRCNN method by changing the preprocessing of Bicubic interpolation with SSI-filtration. We also study and compare the super resolution methods such as transformation technique with SSI filtration technique, SRCNN learning based method and proposed algorithm with the help of Peak-Signal-to-Noise-Ratio (PSNR).

Revised Manuscript Received on August 14, 2019.

Rahul Bhattacharya, Dept. of Electronics and Telecommunication, KIIT University, Bhubaneswar, Odisha, India. (E-mail: rahul.bhattacharya10@gmail.com)

K. Parvathi, Dept. of Electronics and Telecommunication, KIIT University, Bhubaneswar, Odisha, India. (E-mail: kparvati16@gmail.com)

\section{RELATED WORKS}

To improve the resolution or enhancing the image quality there are several methods and most importantly Image Interpolation and Super Resolution are the two methods that usually take under focus. Image Interpolation basically attempts to recover a continuous intensity function from discrete image samples based on a linear deterministic reconstruction $\operatorname{kernel} r(\cdot)$.

$$
\hat{\imath}(\varepsilon)=\sum_{k} c_{k} r_{k}(\varepsilon)
$$

Where $c_{k}$ the interpolation coefficient is determined by input data and $r_{k}(\varepsilon)$ is basically a smooth function such as Spline, Sinc. There are several interpolation techniques such as Bilinear, Bicubic, B-spline, Lanczos which can increase the pixel density without adding the feature-details .But these interpolation techniques are good in case of smooth region and it could not help to distinguish the edge areas from smooth areas [1]. To overcome this problems Li [2] proposed an algorithm called Novel Edge-Directed Interpolation (NEDI) and the main idea of this NADI algorithm is to first calculate coefficients of local covariance from a low resolution image and next employ those estimated co-variances to adapt the interpolation at higher resolution based on the geometric duality between the lowresolution co-variance and the high-resolution co-variance. $\mathrm{Li}$ approaches an image can be modeled as Local Gaussian Field and the local covariance matrix at each pixel is to be computed and after that the interpolation coefficient $c_{k}$ can be calculated adaptively using the principal of Least Square Estimator. D. Su and P. Willis [3] introduces an algorithm is called Data Independent Triangulation which arbitrary use two triangulation to represent four-pixel square mesh. D. Su et al. [3] also used Gouraud Shading to compute pixel value at any point in triangle, which not only improve the model edges but also tends to improve proper interpolation within different geometrical meshes.

Super Resolution (SR) is the process to improve resolution or increase the pixel density in a LR image by using a pair of HR and LR images. The basic difference between Interpolation and SR method is that if a number of input image is one, the image enhancement process is known as Interpolation and if there is more than one input image, the resolution enhancement method would be called as Super Resolution [4]. There are several SR methods to improve resolution and these methods are classified into five basic categories such as (i) Interpolation based, (ii)

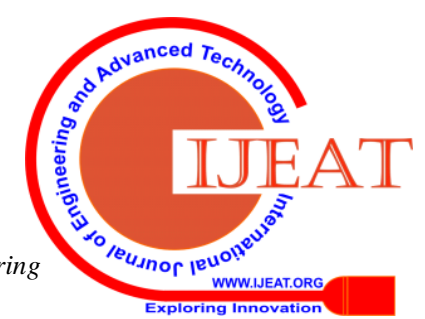


Transformation based, (iii) Reconstruction based, (iv) Filtering based and (v) Learning based. In Interpolation based most of the edge artifacts are being lost and it gets smoothed.

In Transformation Based method the LR images parameters are being transformed to another domain or different basis (like Frequency domain) using Fast Fourier Transformation (FFT) or Walsh Hadamard Transformation (WHT) or Discrete Cosine Transformation (DCT) or Wavelet Transformation [13] to extract the feature details or energy information and increase the resolution by adding the artifact details. William K. Pratt et al. [10] proposed Walsh Hadamard Transformation as a SR transformation based method for feature extraction model and also demands that it gives better performance than Fourier Transformation. P. V. Pithadia et al. [9] approaches Discrete Cosine Transformation (DCT) with Local Binary Pattern (LBP) operator for feature extraction model. Tianton Guo et al. [8] and R. Shivakumar et al. [6] proposed Wavelet Transformation for decomposing the LR images and extract frequency basis details to increase pixel density.

In Reconstruction based approach the desired HR image can be obtained from the relationship between HR and LR images and it also depends on some prior model which can assume the artifacts details of LR image and it can solve the inverse SR problem. Reconstruction based method mainly includes Maximum-a-Posteriori Probability (MAP) method, frequency domain spatial domain algorithm, iterative backprojection method etc. [11]. Minmin et al. [5] proposed a Reconstruction-based Algorithm (RBA) which is depending upon the conditioning of linear-system characterizing the model of degradation and it is analyzed in Fourier domain with the help of perturbation theory. Minmin also proposed a different approach of super resolution in which point spread function (PSF) is taken as an error bound function and it reveals that the flat or blur function can suppress the condition number $(\mathrm{CN})$ of the degradation matrix and the non-integer magnification-factors (MFs) which comes from sampling zero crossing of the Discrete Fourier Transformation of PSF, gives advantage over the integer ones (1s).

Image SR reconstruction methods can be classified into two categories Multi-frame and Single-frame, Multi-frame reconstruction SR method combines the set of multiple frames of LR image of same scene for reconstruction and use iterative back projection method, projection onto convex sets (POCS) method, frequency domain method, MAP method and gives good results. But it has huge consumption of storage and computational complexity. On the other hand, Single-frame reconstruction SR method use single frame of LR image with a single input source. Image interpolation, image scaling, zooming etc. are the example of single-frame SR method. It has less storage consumption and computation complexity but it is inferior to multi-frame reconstruction SR method [12].

In filtering based method the artifact details are being extracted by using a high-pass filter, because most of the feature details are mainly stored in high frequency components. R. Sivakumar et al. [6] approached a filtering technique on a single image super resolution, where input image is first decomposed with wavelet transformation and then calculate the log energies of each decomposed band and next calculate the Scalar Sharpness Index (SSI) and filter the image with SSI parameter which gives a high resolution (HR) image. Sandeep et al. [7] introduces another filtering technique called Block-Based SSI filtering which filters the LR image blocks instead of whole LR image.

Learning based method basically based on machine learning techniques which mainly tries to capture the cooccurrence prior of LR and HR image patches and extract the feature details. Jianchao et al. [15] represent a learning based method using spare representation in terms of coupled- dictionaries jointly trained from LR and HR image-patch pairs. But the dictionary size should be optimized for better results. Detian et al. [14] proposed sparse auto-encoder (SAE) which can boost the stability and accuracy of dictionaries. Detian also introduces zero-phase component analysis (ZCA) whitening method to reduce the redundancy of the joint dictionary set. Chao et al. [16, 17] proposed a deep learning method which directly learns an end-to-end mapping between HR and LR images. The mapping is represented as Convolution-Neural-Network (CNN). Chao also shows later the sparse-code-based SR method with the help of CNN. Chaos's method optimized the hidden layers more accurately and the model they proposed called Super-Resolution Convolution Neural Network (SRCNN).

\section{METHODOLOGY}

\subsection{Filtering based Super Resolution method}

The filtration techniques can be used to improve the resolution of an image. The edge artifacts, intensity details are basically stored in the high-frequency components and the low-frequency components store the texture details. So the filtration technique is employed for filtering out the illposed edge artifacts and intensity details from the LR image. In this paper we are going to discuss about filtration based SR method with SSI parameter.

Firstly a three-level Discrete Wavelet Transformation (DWT) is performed to decompose the LR image and compute the sub-bands of the image namely LL, LH, HL, $\mathrm{HH}$.

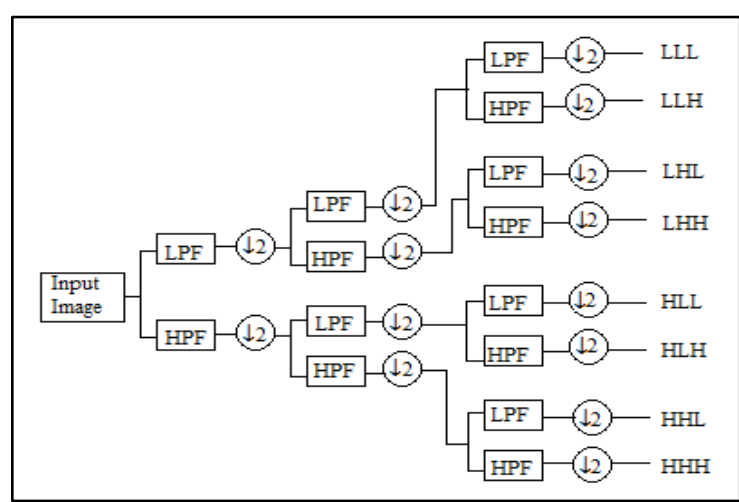

Fig. 1. 3-level DWT decomposition Blue Eyes Intelligence Engineering 
In the next step the log energies of every sub-bands are to be calculated as, [20]

$$
E_{X Y_{k}}=\log _{10}\left(1+\frac{1}{N_{k}} \sum_{i, j} S_{X Y_{k}}^{2}(i, j)\right)
$$

Where, $\mathrm{XY}=\{\mathrm{LH}, \mathrm{HL}, \mathrm{HH}\}, N_{k}, k \in\{1,2,3\}$ is the number of coefficient in sub-bands at level $\mathrm{k}$, and $S_{X Y_{k}}$ is the each decomposition level can be calculated as, [6]

$E=(1-w) *\left(E_{L H}+E_{H L} / 2\right)+w * E_{H H}$

Where $w$ : Weightage value And lastly the Scalar Sharpness Index parameter are to be calculated as, [6]

$$
S S I=\sum_{n=1}^{3} 2^{L-n} E_{n}
$$

In above equation 2.5 , ' $\mathrm{L}$ ' is the factor which is greater than or equal to n. Finally we filter out the high frequency edge artifacts with a multidimensional filter using SSI as a scalar filter parameter which gives a high resolution enlarge output image with high contrast.

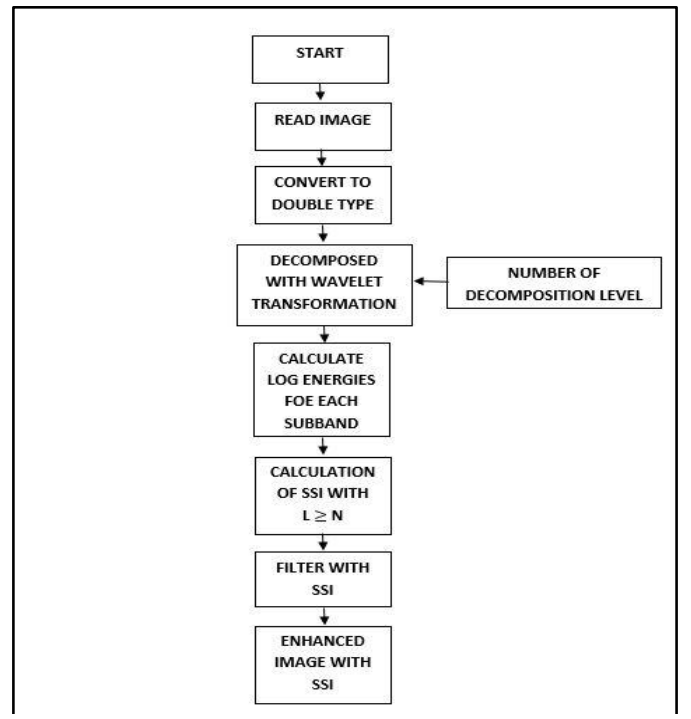

Fig. 2. Flowchart of SSI filtration for SR method

\subsection{Learning based Super Resolution method}

This SR method is a prior model mapping between LR and HR image and this mapping can be done by using any type of neural network such as Feedforward-NeuralNetwork, Recurrent-Neural-Network (RNN), ModularNeural-Network (MNN), Kohonen-Self-Organizing-NeuralNetwork and Convolution-Neural-Network (CNN). In our paper we are going to discuss about the $\mathrm{CNN}$ based learning method for super resolution i.e. SRCNN [16] [17]. This process can be done in three basic steps i.e. (I) Patch extraction, (II) Non-linear mapping, (III) Reconstruction.

(I) Path extraction: In this step we first extract the overlapping patches from the LR image $\mathrm{X}$ and next these patches are represented as high-dimensional vectors which comprise of a set of artifact maps. The number of sets of artifact maps indicates the dimensionality of the vectors. The overlapping patches can be extracted by convolving the LR image with a set of trained filters with a basis. This filters basically implement a set of networks. First layer is formally expressed as an operational function $F_{1}$, [16] subband levels of DWT. Now the weighted log energy for

$F_{1}(X)=\max \left(0, S_{1} * X+A_{1}\right)$

Where [21], $B_{1}$ and $W_{1}$ represents the basis and the filters respectively and ' $*$ ' represents the convolution operator. ' $S_{1}$ ' represents $n_{1}$ filters of size $c \times g_{1} \times g_{1}$, where $g_{1}$ is the size of a filter and c denotes the number of channels lies in input image. ' $S_{1}$ ' performs $n_{1}$ times of convolutions on the LR image and each convolution step holds a karnel size $c \times g_{1} \times g_{1}$. The output is comprised of artifact maps and $A_{1}$ is an $n_{1}$ - dimensional vector. A filter is connected with each element of $n_{1}$ - dimensional vector. RectifiedLinear-Unit ( ReLU ) [18] is applied on the filter response.

(II) Non-linear mapping: In that operation the highdimensional vector maps onto another highdimensional vector. These mapped-vectors are basically the high-dimensional patch which comprise another set of artifact maps. Here we basically map each $n_{1}$-dimensional vectors onto $n_{2^{-}}$dimensional vectors. In this case the patches are the convolved filter extracted patch of the artifact map. Second layer operation can be expressed as $F_{2}$, [16]

$$
F_{2}(X)=\max \left(0, S_{2} * X+A_{2}\right)
$$

Here [21], $S_{2}$ consist of $n_{2}$ filters of size $n_{2} \times g_{2} \times g_{2}$, and $A_{2}$ is a $n_{2^{-}}$dimensional-basis. Each output is a $n_{2}$ dimensional-vector which represents high-resolution-patch that will be employed for reconstruction. To increase the nonlinearity it is possible to add more convolution layer.

(III) Reconstruction: The above high-resolution patches are aggregated in this operations to produce the final highresolution image. It is expected that this image must be matched with the ground truth Y. Mostly the resultant high resolution images are generated by averaging the predicted overlapping high-resolution patches. A set of pre-defined filters on a feature map is considered as a tool for averaging where each position of the 'flattened' vector is basically represents a high-resolution-patch. Third convolution layer can be formulated as, [16]

$F_{3}(X)=S_{3} * F_{2}(X)+A_{3}$

Where, $S_{3}$ represents c number of filters supports $n_{2} \times$ $g_{3} \times g_{3}$ and $A_{3}$ is a c-dimensional-basis-vector.

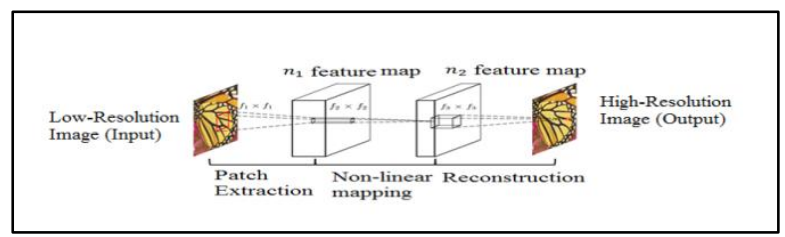

Fig. 3. Methodical diagram of learning based SR method (SRCNN) with 3 layers

\subsection{Proposed algorithm for Super Resolution method}

The proposed algorithm is basically generated to improve the SRCNN methodology. In SRCNN technique the low resolution image is initially interpolated by bicubic interpolation method for enlargement which is named as a preprocessing technique of learning based SR method. Now 
as we know that the interpolation technique smoothen the low resolution image artifacts, so if the filtration technique is used as a preprocessing technique instead of only using interpolation technique, the output will give a high resolution image with better artifact details.

\begin{tabular}{|c|c|c|c|c|c|}
\hline $\begin{array}{l}\text { Low } \\
\text { Resolution } \\
\text { Image }\end{array}$ & $\begin{array}{l}\text { Resize the } \\
\text { image with } \\
\text { interpolation } \\
\text { technique }\end{array}$ & & $\begin{array}{l}\text { Perform SSI- } \\
\text { filtration } \\
\text { method }\end{array}$ & $\rightarrow$ SRCNN & $\begin{array}{l}\text { High } \\
\text { Resolution } \\
\text { Image }\end{array}$ \\
\hline
\end{tabular}

Fig. 4. Processing step block diagram of proposed algorithm

\section{RESULTS AND DISCUSSION}

In this paper we use MATLAB, 2017 software to execute our methodologies. In case of our study we consider two parameters Peak-Signal-to-Noise-Ratio (PSNR) to compare the qualitative improvement of LR image after super resolution.

In SSI filtration technique we will be taking the weightage $(w)$ and ' $\mathrm{L}$ ' parameter accordingly, which is the best suited value for a specific LR image. In case of SRCNN methodology we have taken a filter setup specification as $g_{1}=9, g_{2}=5, g_{3}=5, n_{1}=64, n_{2}=32$ and convolution biases as $a_{1}=64 \times 1, a_{2}=32 \times 1, a_{3}=1 \times 1$. [16][17]

In our paper we choose nine different types of pictures and execute the above mentioned methodologies for super resolution in order to get a HR enlarged output image. We perform the methodologies in different image patches with an upscaling factor of four (4) for enlargement.

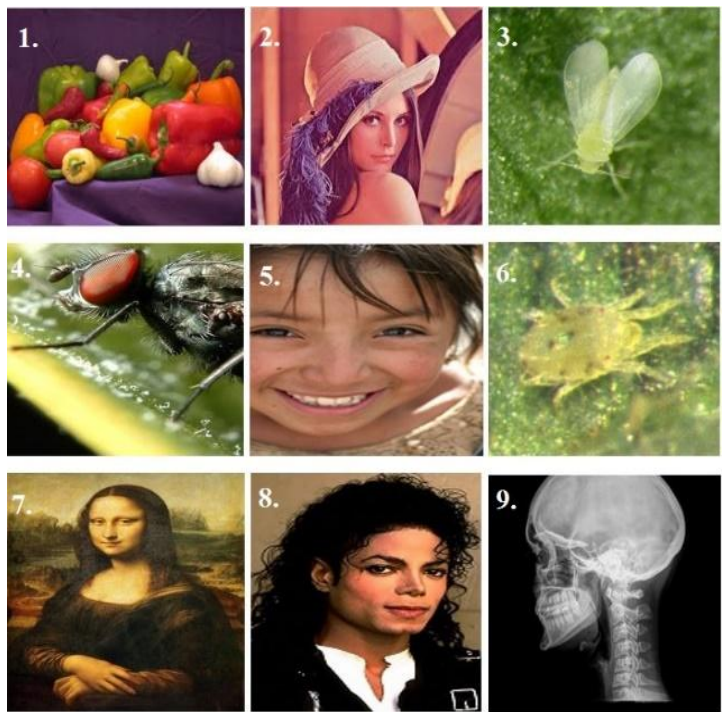

Fig. 5. Input Low Resolution Image set

Now for different SR methodologies the nine picture patches are giving results as,
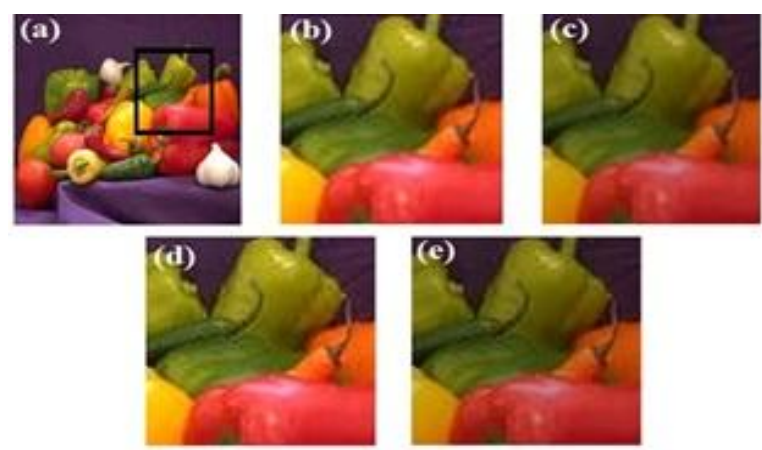

Fig. 5.1. (a) Original image with patch size $79 \times 70$ pixels, (b) Interpolated image, (c) SSI filtered image (W=0.8 and $\mathrm{L}=7$ ), (d) SRCNN output image, (e) Proposed algorithm output image.

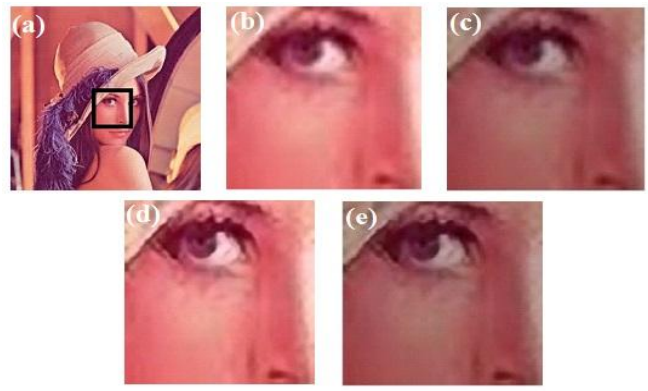

Fig. 5.2. (a) Original image with patch size $38 \times 38$ pixels, (b) Interpolated image, (c) SSI filtered image (W=0.8 and $L=6$ ), (d) SRCNN output image, (e) Proposed algorithm output image.
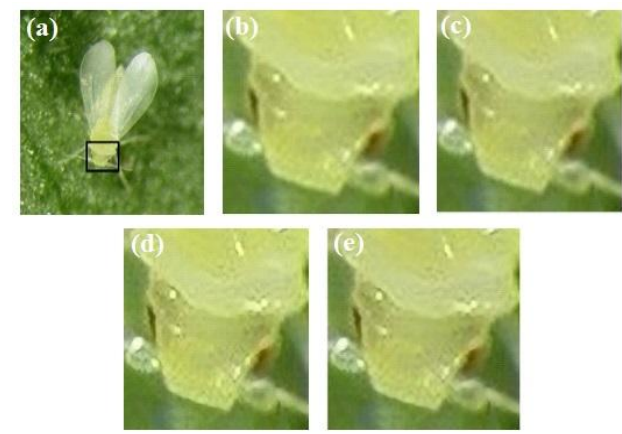

Fig. 5.3. (a) Original image with patch size $75 \times 56$ pixels, (b) Interpolated image, (c) SSI filtered image (W=0.8 and $\mathrm{L}=8$ ), (d) SRCNN output image, (e) Proposed algorithm output image.

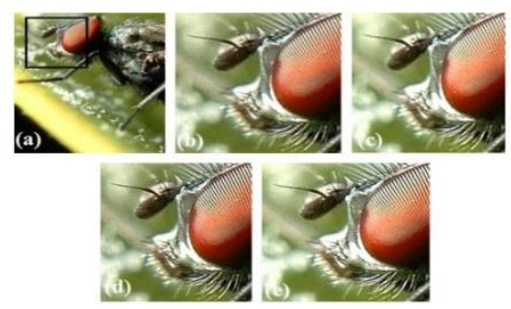

Fig. 5.4. (a) Original image with patch size 114x131 pixels, (b) Interpolated image, (c) SSI filtered image (W=0.9 and $\mathrm{L}=3$ ), (d) SRCNN output image, (e)

Proposed algorithm output image

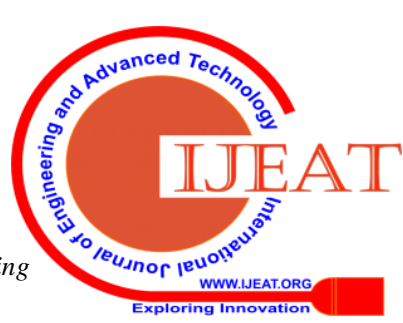



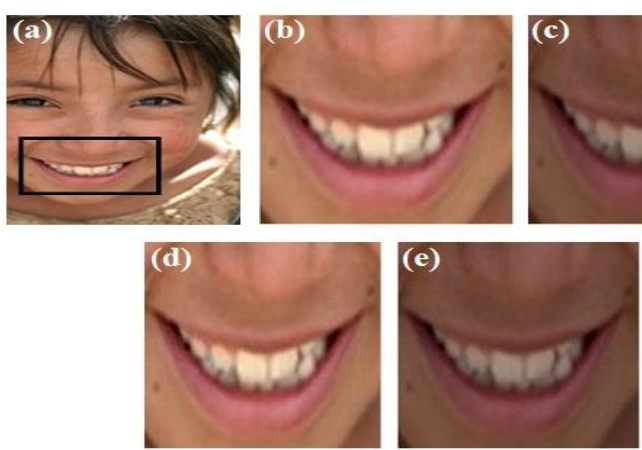

Fig. 5.5. (a) Original image with patch size $131 \times 83$ pixels, (b) Interpolated image, (c) SSI filtered image (W=0.8 and $L=10$ ), (d) SRCNN output image, (e) Proposed algorithm output image.
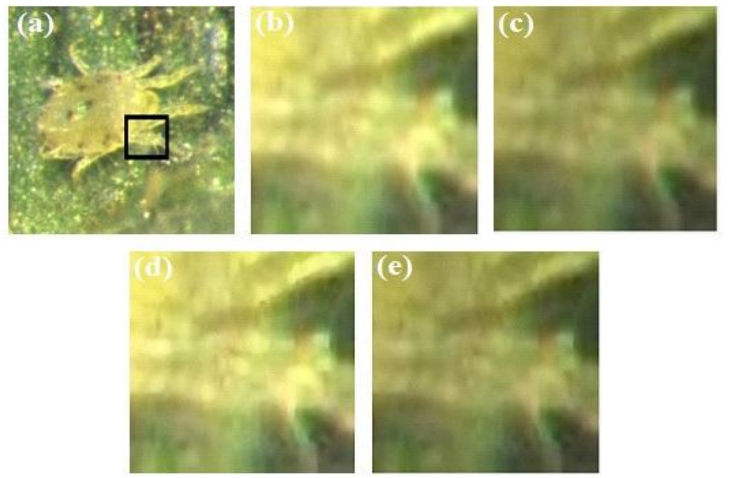

Fig. 5.6. (a) Original image with patch size $52 \times 36$ pixels, (b) Interpolated image, (c) SSI filtered image (W=0.8 and L=9), (d) SRCNN output image, (e) Proposed algorithm output image.
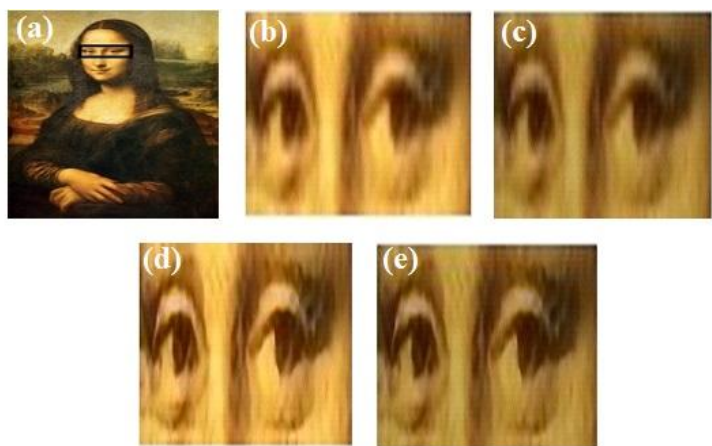

Fig 5.7. (a) Original image with patch size $67 \times 16$ pixels, (b) Interpolated image, (c) SSI filtered image (W=0.9 and $L=7$ ), (d) SRCNN output image, (e) Proposed algorithm output image.
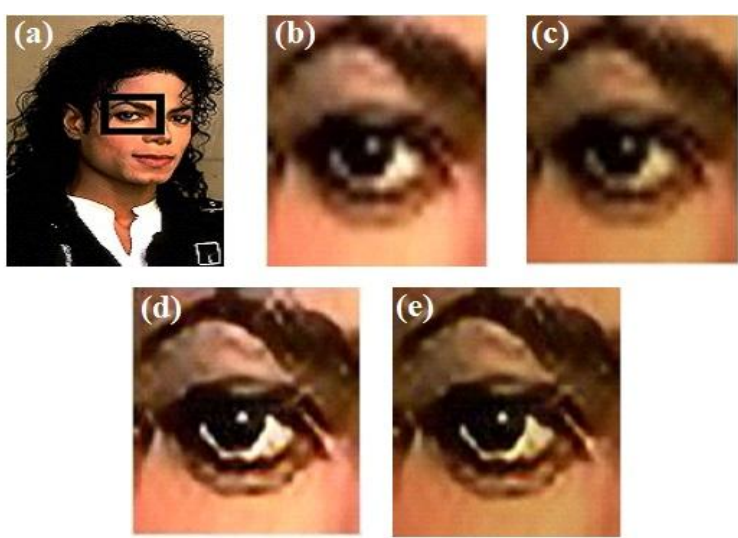

Fig. 5.8. (a) Original image with patch size $27 \times 20$ pixels, (b) Interpolated image, (c) SSI filtered image (W=0.8 and $\mathrm{L}=5$ ), (d) SRCNN output image, (e) Proposed algorithm output image.
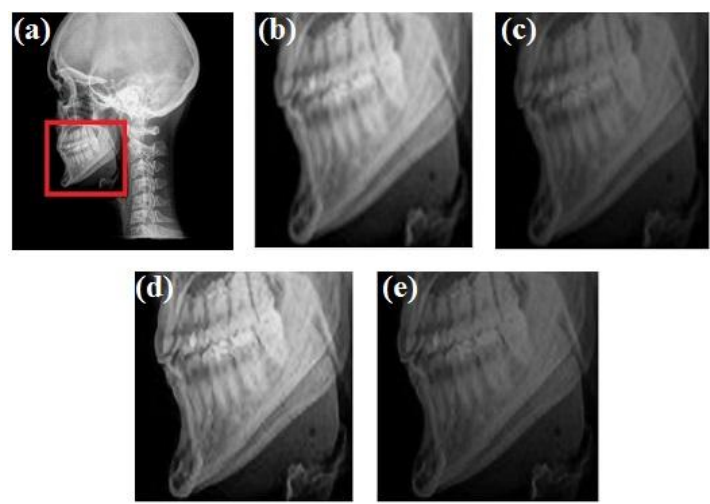

Fig. 5.9. (a) Original image with patch size $81 \times 67$ pixels, (b) Interpolated image, (c) SSI filtered image ( $W=0.8$ and $\mathrm{L}=6$ ), (d) SRCNN output image, (e) Proposed algorithm output image.

Table 1. PSNR Comparison Table

\begin{tabular}{|c|c|c|c|}
\hline IMAGES & $\begin{array}{l}\text { SSI- } \\
\text { FILTRATION }\end{array}$ & SRCNN & $\begin{array}{l}\text { PROPOSED } \\
\text { ALGO. }\end{array}$ \\
\hline $\begin{array}{l}\text { Fruit } \\
(79 \times 70)\end{array}$ & 70.2031 & 89.3158 & 91.6950 \\
\hline $\begin{array}{l}\text { Lena } \\
(38 X 38)\end{array}$ & 65.2271 & 83.1984 & 86.5110 \\
\hline $\begin{array}{l}\text { White Fly } \\
\text { (75X56) }\end{array}$ & 84.2889 & 89.1451 & 89.5944 \\
\hline $\begin{array}{l}\text { Fly Body } \\
(114 X 131)\end{array}$ & 61.1170 & 72.8811 & 77.8345 \\
\hline $\begin{array}{l}\text { Child } \\
(131 \times 83)\end{array}$ & 64.3273 & 101.0842 & 105.3360 \\
\hline $\begin{array}{l}\text { Leaf Insect } \\
(52 \times 36)\end{array}$ & 64.7019 & 92.7475 & 97.3267 \\
\hline $\begin{array}{l}\text { Monalisa } \\
(67 X 16)\end{array}$ & 67.2601 & 81.4415 & 84.1341 \\
\hline $\begin{array}{l}\text { Michael } \\
(27 X 20)\end{array}$ & 70.4698 & 73.0793 & 74.8079 \\
\hline $\begin{array}{l}\text { Human Skull } \\
(81 \times 67)\end{array}$ & 62.6069 & 85.4084 & 91.7156 \\
\hline
\end{tabular}

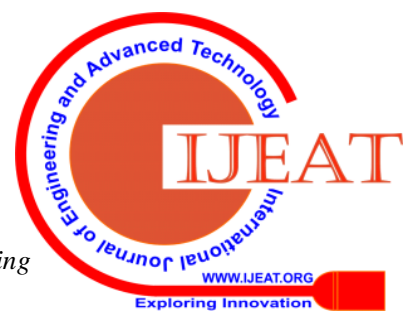


The above results shows that the original small image patches are enlarged with a scaling factor 4 and the outputs are the high-resolution image patches. The results of proposed methods are little dark in compare to others because some of the averaged or predicted pixel values of enlarged image is replaced by 255 after filtration. The comparison table of PSNR basically computed with respect to the enlarged interpolated image for SSI-Filtration and SRCNN methods. And for our proposed algorithm we calculate the PSNR w.r.t the preprocessing output value i.e. the output value of SSI filtration.

\section{CONCLUSION}

Our paper presents a thorough overview of super resolution methodologies which has been proposed for several years. We also approach a new algorithm to enhance the usefulness of Super-resolution Convolution Neural Network (SRCNN) technique. The proposed algorithm shows a better result in compare to SRCNN with interpolation as a preprocessing technique. It is also noticeable that with a proper SSI filtration parameter value a low resolution image can be enhanced with better artifact and intensity details. For image 4 we got the best PSNR value of 105.33 .

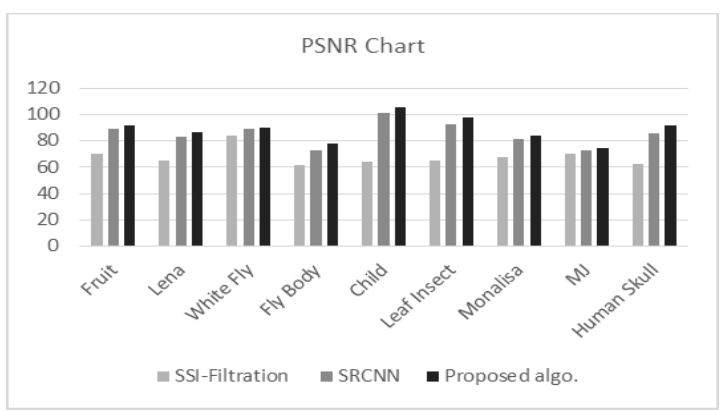

Fig. 6. PSNR Chart of nine images

\section{REFERENCES}

1. Xin Ye, Xiqun Lu, 'A Performance of Image Interpolation and Super Resolution Algorithm', ICMT, pp. 4776-4779, 2011.

2. Xin Li, M. T. Orchad, ' New Edge-Directed Interpolation', IEEE, Vol. 10, No. 10, 1057-7147, Oct. 2001.

3. Jun Yi Duan, Cheng Ming Liu, Zhi Hui Yue, 'Image Interpolation by Pixel-level Data-Dependent Triangulation on Android Platform', CSMA, 2017.

4. S. Baker, T. Kanade, 'Limits on Super-resolution and How to Break Them', IEEE Trans., On Pattern Analysis and Machine Intelligence, 24(9): 1167-11, 2002.

5. Minmin Shen, Ci Wang, Ping Xue, Weisi Lin, 'Performance of Reconstruction-based Super-resolution with Regularization', Elsevier Ins., 640-650, 2010.

6. Venkatesan, C., P. Karthigaikumar, and S. Satheeskumaran. "Mobile cloud computing for ECG telemonitoring and realtime coronary heart disease risk detection." Biomedical Signal Processing and Control 44 (2018): 138-145.

7. Sandeep Mishra, R. Patra, A. Pattanayak, S. Pradhan, 'Block Based Enhancement of Satellite Image Using Sharpness Index Filtering', IOSR-JECE, ISSN, Vol. 8, pp. 20-24, Nov.- Dec. 2013.

8. Tingtong Guo, H. S. Mousani, T. H. Vu, Vishal Monga, 'Deep Wavelet Prediction for Image Super-Resolution', IEEE Xplore, 24th Aug., 2017.
9. P. V. Pithadia, P. P. Gajjar, J. V. Dave, 'Super-resolution Using DCT Based Learning with LBP as Feature Model', IEEE, ICCCNT'12, 28th Jul., 2012.

10. Willium K. Pratt, Julius Kane, H. C. Andrew, 'Hadamard Transform Image Coding', IEEE, Vol. 57, No. 1, Jan. 1969.

11. Yaolan Zhang, Yijun Liu, 'Single Image Super-resolution Reconstruction Method Based on LC-KSVD Algorithm', AIP, 2017.

12. Noriaki Suetake, M. Sakano, E. Uchino, 'Image Super Resolution Based on Local Self-Similarity', OPTICAL REVIEW, Vol. 33, No. 7, Nov. 2011.

13. Mathew k, Dr. S. Shibu, 'Wavelet Based Technique for Super Resolution Image Reconstruction', International Journal of Computer Applications, Vol. 33, No. 7, Nov. 2011.

14. Detian Huang, W. Huang, Z. Yuan, Yanming Lin, J. Zheng, L. Zheng, 'Image Super Resolution Algorithm Based on an Improved Sparse Autoencoder', MDPI, 5th Jan, 2018.

15. Jianchao Yang, Jhon Wright, Thomas S. Hung, Yi Ma, 'Image Super Resolution via Sparse Representation', IEEE Trans. On Image Processing, Vol. 19, No. 11, Nov. 2010.

16. Chao Dong, Chen Change Loy, K. He, X. Tang, 'Image Super Resolution Using Deep Convolution Network', IEEE, 31st Jul. 2015.

17. Chao Dong, Chen Change Loy, K. He, X. Tang, 'Learning a Deep Convolution Network for Image Super Resolution', ECCV, pp. 184-199, 2014.

18. Venkatesan, C., P. Karthigaikumar, Anand Paul, S. Satheeskumaran, and R. Kumar. "ECG signal preprocessing and SVM classifier-based abnormality detection in remote healthcare applications." IEEE Access 6 (2018): 9767-9773.

19. "Digital Image Processing Using MATLAB (Second Edition)", Rafel C. Gonzalez, Richard E. woods and Steven L. Eddins, 2003.

20. Phong Van $\mathrm{Vu}$, 'ON THE USE OF IMAGE SHARPNESS MEASURE TO JPEG2000 NO-REFERENCE IMAGE QUALITY ASSESSMENT', Oklahoma State University, M.S Thesis, December, 2013.

21. Wenming Yang, Xuechen Zhang, Yapeng Tian, Wei Wang, Jing-Hao Xue, 'Deep Learning for Single Image SuperResolution: A Brief Review', arXiv:1808.03344v1, 9 Aug 2018. 